topography of corrosion ${ }^{6}$. $M$. Canac has utilized an optical method to investigate the character of the attack on a corroded surface; briefly, the method consists in studying the distribution in space of the light diffused from a corroded surface, the angle of incidence being varied. He has shown by mathematical analysis that the diffusion/time curves at different angles of incidence should be characteristic for different types of attack, for example, for uniform corrosion spreading outwards at a constant rate from a regularly distributed number of centres, for intercrystalline corrosion, etc., and he has observed close approximations to the theoretical curves in actual corrosion experiments. This method and also one described by $M$. Nicolau for studying the roughness of a surface, depending on observations of the rate of escape of compressed air from a standard orifice brought into close proximity with it, should prove of value in the study of certain types of corrosion problems.

Prof. A. M. Portevin and Dr. E. Herzog communicated the results of some tests on the corrosion of a low alloy chromium-aluminium steel exposed to sea air and to immersion in sea-water at five different ports in France and Algeria?. It is encouraging to note that the results showed a marked superiority in the behaviour of the low alloy steel as compared with ordinary steels, not only in the atmospheric but also in the immersion tests. This observation may be of considerable practical importance, since the improvements effected so far in the corrosion resistance of steel by the addition of small amounts of alloying elements, without markedly increasing its cost, have not proved nearly so pronounced in the case of exposure to immersion in sea-water as in that of exposure to atmospheric corrosion.
Prof. G. Chaudron gave a general survey of his researches at Lille, which deal both with ferrous and with light alloys, and stressed the fact that studies of dissolution potential should include the behaviour not only of the bare metal but also of the metal covered with its oxide film. Prof. A. Travers of Nancy communicated the results of an examination of a cast iron pipe that had suffered severe graphitiza. tion whilst lying in the soil, and concluded, as is also the view of Dutch experts who have studied the problem, that bacteriological action plays an im. portant part in this phenomenon; the reduction of calcium sulphate in the soil as a result of bacterio. logical action also formed the subject of a paper by Prof. R. O. Rotini, of Milan. It is also possible that an electrolytic method of polishing metals, devised by $M$. P. A. Jacquet ${ }^{8}$ which, the author states, does not result in the formation of a Beilby layer or mechanical disturbance of the metal, may have other interesting applications besides its effect on the structure of electro-deposits, which he discussed.

It remains to add that the arrangements of the Conference were in the capable hands of $M$. J. Desthieux, the secretary of the Académie Méditér. ranéenne, that thirty papers were read or presented and that at the end of the Conference the proceedings were very ably summarized by $M$. Canac, who acted as rapporteur.

${ }^{1}$ Grard, C., Méteux, 9, 291 (1934).

${ }^{2}$ Legendre, R., Recherches et Inventions, 17, 29 (1937).

${ }^{3}$ Veillon, R., Annales de l'Institut Technique du Bâtiment et des Travaux Publics, 1, 19 (1936).

1 Vila, A., Recherches et Inventions, 17, 79 (1936).

${ }^{5}$ Roux, J. Métaux, 10, 509 (1935).

${ }^{6}$ Canac, F., C.R., Jan. 3, 1933; Nov. 19, 1934; July 29, 1935

'Portevin, A. M., and Herzog, T., C.R., Dec. 14, 1936.

8 ef. Nature, 135, 1076 (1935).

\title{
The Post Office Speaking Clock in Great Britain
}

$\mathrm{U}^{\mathrm{P}}$ to the beginning of the nineteenth century the time in most towns was taken from public clocks of various kinds, which occasionally varied appreciably from one another. This was a serious obstacle to the postal services, and led to the practice on mail coaches and trains of carrying chronometers to synchronize local post office clocks with a standard clock in London. Shortly after the introduction of the electric telegraph, electro-mechanical devices of various kinds were controlled by telegraphed signals. But it was not until 1874, a few years after the Government acquired the telegraph system, that successful distribution of the 10.00 a.m. Greenwich time signal was accomplished over sixty different lines. The development of this service into the very accurate International Time Signal transmitted from Rugby radio station at 10.00 and 18.00 G.M.T. daily was made in 1927 . This service has the disadvantage of not always being available in the home. The well known six 'pips' of the B.B.C. have the disadvantage of being only available at certain times. Then came the synchronous motor-clocks running on frequency-controlled mains, but at present these clocks are far from being in universal use.

The success of 'speaking clocks' abroad, especially in Paris, encouraged the General Post Office to design a speaking clock service which would be of use to every house or shop connected with the P.O. telephones. It was decided that the inaccuracy of the clock should not exceed a tenth of a second, fast or slow, and that the time should be announced six times a minute, each announcement being followed by three audio-frequency pips, the last of which gives the exact time spoken. To hear the time a subscriber connected to an automatic exchange dials the code TIM, and one connected to a manual exchange asks for 'Time'.

A technical paper on this novel service was read to the Institution of Electrical Engineers on December 3 by Dr. E. A. Speight and O. W. Gill. They pointed out that when the exchange is called at, say, 10.25, a voice is heard saying, "At the third stroke it will be ten twenty-five and ten seconds". The third of the three pip sounds gives this time precisely. The next announcement will be ". . . . ten twenty-five and twenty seconds", and so on. Most recording systems may be classified as mechanical, magnetic or photographic in principle. The gramophone is typical of the instruments which give a mechanical record. It is compact and cheap to produce, but it has a fairly high noise level which increases steadily with the fairly rapid wear occurring in reproduction. Experiments on gramophone records made in India showed that it was necessary to replace them in one or two days time. Magnetic sound recording is done by means of a steel tape, but owing to its high velocity a considerable length of tape is required. In this case the initial quality 
of the reproduced sound is good, but it deteriorates rapidly. The system used by the Post Office is the photographic system, and photocells are used. The technique is complicated, but the record is not subjected to any mechanical wear and is therefore, for all practical purposes, permanent.
During the first week of the public operation of the service, nearly 400,000 calls were made, and during each subsequent week the number has remained practically constant at about 200,000 . The relay sets at present permanently installed can each supply 100 simultaneous calls.

\section{Noise on the Road}

$\mathrm{T}$ HE Departmental Committee on "Noise in the Operation of Mechanically Propelled Vehicles" has published its third interim report (H.M. Stationery Office, $1 s$. net). The Committee, of which Dr. G. W. C. Kaye is chairman, was set up by the Minister of Transport in 1934. The present report is based on loudness tests carried out for the Committee by the National Physical Laboratory on some 600 'used' motor-vehicles representative of all the main types on the roads to-day, and of various ages up to thirteen years. A comparison of the results with those from the earlier tests on new vehicles, shows that on the average the used vehicles were the louder by some $4-5$ phons. In the range of noise met with on the road (about 70-105 phons) ordinary motorcars were much the quietest vehicles, while amongst the loudest were certain motor-cycles and sports cars, particularly at high speeds.

The noise from the engines of ordinary cars and commercial vehicles is louder than the exhaust noise, but with motor-cycles and sports cars the position is reversed. This fact lends support to the Committee's earlier criticism of the unsatisfactory nature of the silencing of many motor-cycles and sports cars, and the investigation generally indicates that greater progress has been made in the silencing of sports cars than of motor-cycles.

In its previous reports the Committee made the recommendation that new motor-vehicles should not be offered for sale if the loudness under prescribed conditions of test exceeded 90 phons, adding that motor-cycles should be allowed a latitude of 5 phons for two years. The Committee now proposes for vehicles actually in use on the road a limit of 95 phons (that is, about the noise in a tube train). No vehicles would be allowed on the highway if the loudness, when measured at a point $18 \mathrm{ft}$. laterally from the middle of the vehicle or $25 \mathrm{ft}$. behind the open end of the exhaust pipe, exceeded this limit in a normal running test and in a racing engine test conducted in the same way as for newly manufactured vehicles. The Committee also recommends that this limitation of loudness should be applied at first only to vehicles first registered after an appointed day, but that two years thereafter it should apply to vehicles of any age.

It will be appreciated that the noise limit proposed, while making very modest demands on the majority of manufacturers and owners of motor-vehicles, would, by ruling out the arch offenders, form a substantial contribution to the amenities of the road. It is to be hoped, moreover, in the public interest that future developments will enable the limit to be lowered as time goes on. At the Minister's request, the Committee is now investigating the noise caused by motor horns with the view of advising him whether action might usefully be taken to abolish the more strident noises of this kind. The question of the noise arising from trams and trolley buses is under examination by the Ministry in conjunction with the associations concerned.

\section{Hirosima Wave Geometry and Field Theory}

$\mathrm{R}$ EFERENCE has already been made in this journal (Dec. 21, 1935) to the vigorous school of mathematical physics now flourishing in the Hirosima University, Japan. These investigations are continuing (J. Sci. Hirosima Univ., A, 7 ; 1937), bringing the number of memoirs on the subject up to fiftcen. A good summary of the whole series is contained in the paper "Geometrization of the Law of Physics" (ibid., p. 81) by Y. Mimura.

It is claimed that the new theory bears a relation to Einstein's theory similar to that which Einstein's bears to Newton's, or to that which modern quantum mechanics bears to the old quantum theory. Einstein's theory is described as semi-geometrical, and the same term is applied to all existing unified field theories, as they are all built by a mixture of two procedures, namely, the finding of an appropriate geometry, and the finding of an appropriate invariant density to be used with a generalized Hamilton's principle, which is assumed to be the funda. mental law of physics.

The Hirosima school considers that the generalized
Hamilton's principle should be dispensed with, and they replace it by the condition of the complete integrability of the fundamental differential equation, which is obtained by purely geometrical means. In the hope that 'wave geometry' is identical with physics, extensive investigations of possible varieties have been carried out, including those corresponding to four-dimensional ordinary metrical geometry, to projective geometry, and to conformal geometry.

It is acknowledged that the question as to which wave geometry is to be identified with physies can only be judged by its results. The new field theory has already succeeded in deducing some important physical results, such as a gravitional wave in Maxwellian form, a line element of de Sitter's form, and one equivalent to Schwarzschild's. Perhaps it is not unfair to say that, in these days of competing unified field theories, the ordinary physicist must be allowed to suspend judgment until the physical consequences of the theories have been worked out very much more fully than has been done at present. 